\title{
Predictive Factors Associated with the Success of Pneumatic Dilatation in Japanese Patients with Primary Achalasia: A Study Using High-Resolution Manometry
}

\author{
Hiroshi Yamashita Kiyoshi Ashida Takumi Fukuchi Yoshiaki Nagatani \\ Hideaki Koga Kasane Senda Takaaki Eguchi Satoshi Ubukata \\ Shinpei Kawaguchi Aya Ueda Toshio Tanaka Rina Ohashi Dai Ito \\ Department of Gastroenterology and Hepatology, Saiseikai Nakatsu Hospital, Osaka, Japan
}

\section{Key Words}

Primary achalasia $\cdot$ Pneumatic dilatation $\cdot$ High-resolution

manometry $\cdot$ New classification of achalasia

\begin{abstract}
Background/Aims: A new classification of achalasia using high-resolution manometry (HRM) has recently been suggested. Pneumatic dilatation (PD) is a common treatment for primary achalasia. The usefulness of the new classification and HRM for the treatment and follow-up of patients after $P D$ is unknown. The aim of this study was to evaluate the PD effectiveness and the predictive factors of success in Japanese patients with achalasia using HRM and the new classification of achalasia. Methods: Twenty-five patients were diagnosed with primary achalasia using HRM and treated by PD in our hospital. We evaluated symptom scores and esophageal manometry 6 and 12 months after the first PD. Results: After the first PD treatment, remission occurred in 24 out of 25 (96.0\%) patients at 6 months and in 19 out of 25 (76.0\%) patients at 12 months. With the new classification of achalasia, the success rates were $83.3,80.0$ and $50 \%$ for types I, II and III, respectively, 12 months after PD. The median age of the successful group was significantly greater than that of
\end{abstract}

the failure group (47.1 vs. 37.0 years, $p<0.05$ ). The median residual lower esophageal sphincter (LES) pressure 6 months after PD in the successful group was significantly lower than that of the failure group ( 9.0 vs. $15.5 \mathrm{~mm} \mathrm{Hg}, \mathrm{p}<0.05$ ). Conclusion: Good predictors of PD success were old age ( $>40$ years) and residual LES pressures less than $15 \mathrm{~mm} \mathrm{Hg} 6$ months after PD.

Copyright $\odot 2013$ S. Karger AG, Basel

\section{Introduction}

Achalasia is a primary motility disorder of the esophagus that is characterized by a loss of peristalsis in the esophageal body, impaired relaxation of the lower esophageal sphincter (LES) during swallowing and increased resting pressure of the LES. It commonly produces dysphagia, regurgitation of undigested food and chest pain. The underlying pathological state is a loss of ganglion cells in the myenteric plexus, but the precise etiology remains unknown [1]. Achalasia is a rare disease, with an estimated annual incidence of 1 case per 100,000 people [2].

The aim of therapy in patients with achalasia is to reduce distal obstructions that can be treated by pneumat-

\section{KARGER}

Fax +4161306 1234

E-Mail karger@karger.ch

www.karger.com (c) 2013 S. Karger AG, Basel

0012-2823/13/0871-0023\$38.00/0

Accessible online at:

www.karger.com/dig
Hiroshi Yamashita

Department of Gastroenterology and Hepatology Saiseikai Nakatsu Hospital

2-10-39 Shibata, Kita-ku, Osaka 530-0012 (Japan)

E-Mail onakaryoko0310@gmail.com 
ic dilatation (PD) or surgical esophagomyotomy [3]. In the modern era, endoscopic therapies have been simplified by the advent of the Rigiflex Microinvasive Balloon (Boston Scientific, Watertown, Mass., USA), and myotomy has evolved from the open thoracotomies of 20 years ago to the current standard of laparoscopic abdominal myotomy. Most gastroenterologists use PD as a first-line treatment because they consider this option to have a lower risk of morbidity and mortality. PD can result in remission in $67-90 \%$ of patients [3]. Tanaka et al. [4] reported that old age was the only independent factor that was associated with the success of PD. In addition, Eckardt et al. [5] showed that older age and lower post-therapy LES pressures were predictors of good treatment outcomes in patients treated with PD. In Japan, however, there are few studies of PD in patients with primary achalasia.

It has been suggested that the use of objective testing, such as esophageal manometry, could be useful for guiding treatment $[6,7]$. High-resolution manometry (HRM) is the most accurate method that can evaluate esophageal motility. However, the usefulness of HRM during followup of post-PD patients has not been explored. Recently, Pandolfino et al. [8] reported that achalasia could be categorized into 3 subtypes by HRM. The 3 subtypes are distinct in terms of their responsiveness to treatments and, therefore, the new classification of achalasia may be clinically useful for predicting the efficacy of treatment. The aim of this study was to evaluate the effectiveness of $\mathrm{PD}$ and its predictive factors in Japanese patients with achalasia using HRM and the new classification of achalasia.

\section{Methods}

From July 2005 to January 2011, 25 consecutive patients with achalasia were invited to take part in the study. All patients were clinically assessed before the PD, with gastrointestinal endoscopy, barium esophagrams and esophageal manometry. Exclusion criteria were a previous PD and any previous esophageal or gastric surgery. Patients were not treated with any drugs that could alter the PD results.

This observational study was carried out in Saiseikai Nakatsu Hospital. The study was conducted in accordance with the Declaration of Helsinki, the consolidated Good Clinical Practice guidelines and the applicable regulatory requirements.

\section{Esophageal Manometry}

An HRM assembly with 36 solid-state sensors that were spaced at $1-\mathrm{cm}$ intervals (ManoScan360, Sierra Scientific Instruments LLC, Los Angeles, Calif., USA) was used on all patients before the PD. Each sensor was circumferentially sensitive and zeroed to gastric pressure. The HRM assembly was passed transnasally and positioned in order to record from the hypopharynx to the stomach with about 5 intragastric sensors. Studies were performed while the patient was in a sitting position after at least a 6-hour fast. The manometric protocol included a 5 -min period to assess basal sphincter pressure and ten 5-ml water swallows.

\section{Manometry Analysis}

All manometric analyses were done using ManoView software (Sierra Scientific Instruments LLC), which was applied to the data tracings that were viewed in the color pressure topography mode. Impaired esophageal-gastric junction relaxation was defined as an average 4-second integrated relaxation pressure that was greater than $15 \mathrm{~mm} \mathrm{Hg}$ [9]. Pandolfino et al. [8] reported that achalasia could be categorized into 3 subtypes by HRM. In type I achalasia, there is no distal esophageal pressurization greater than $30 \mathrm{~mm}$ $\mathrm{Hg}$ in more than 8 of the 10 test swallows (fig. 1). In type II achalasia, at least 2 test swallows are associated with panesophageal pressurization that is greater than $30 \mathrm{~mm} \mathrm{Hg}$ (fig. 2). Type III patients have 2 or more spastic contractions with or without periods of compartmentalized pressurization (fig. 3).

\section{Pneumatic Dilation}

All PDs were performed using Rigiflex balloon dilators with fluoroscopic control. The first PD was usually performed with a $3.0-\mathrm{cm}$ balloon. Patients fasted for at least $12 \mathrm{~h}$ before the procedure. All patients were sedated with intravenous midazolam and pethidine hydrochloride. After the gastrointestinal endoscopy, the Rigiflex dilator was passed over a guide wire and the balloon was positioned across the diaphragmatic hiatus using the radiopaque markers as guides. The balloon was then inflated until the waist was completely obliterated and the inflation was maintained for $60 \mathrm{~s}$. The balloon was inflated 3 times at 60 -second intervals. A gastrografin swallow was performed immediately after dilation in order to identify any esophageal perforations. All patients were hospitalized for at least 1 day post-dilatation for observation of possible complications, including retrosternal pain, perforation, bleeding, fever or vomiting. Normal diet and activity were commenced after $24 \mathrm{~h}$ in the absence of signs of the abovementioned complications. If symptoms were not relieved after $\mathrm{PD}$, we performed a second treatment using a $3.5-\mathrm{cm}$ balloon within a week.

\section{Clinical Evaluation}

We evaluated the main symptoms and LES pressures at 6 and 12 months after the first PD. At the initial investigation and at each subsequent visit, the main symptoms (dysphagia and chest pain) were evaluated and each was given a score between 0 and 2 (almost no symptoms $=0$, occasional symptoms $=1$, daily symptoms $=2$ ). Patients were considered to be in clinical remission when the total scores (0-4) were less than 2 and each symptom was less than 1 . We evaluated the success ratio of PD after 6 and 12 months, respectively. Those patients in clinical remission with a follow-up period shorter than 1 year were excluded from this analysis.

\section{Statistical Analysis}

Comparisons of the manometry data and the demographic data were performed using the Mann-Whitney test. $p$ values less than 0.05 were considered statistically significant. 
Table 1. Demographic characteristics of 25 patients with achalasia according to its new classification

\begin{tabular}{lccc}
\hline & Type I $(\mathrm{n}=6)$ & Type II $(\mathrm{n}=15)$ & Type III $(\mathrm{n}=4)$ \\
\hline Age, years & $43.6(23-75)$ & $51.5(25-84)$ & $60.2(23-88)^{*}$ \\
Gender (male/female) & $2 / 4$ & $7 / 8$ & $3 / 1$ \\
Duration of symptoms, years & $9.5(1-30)$ & $5.3(1-10.5)$ & $6.7(1.5-11.5)$ \\
Symptom score & $2.8(2-4)$ & $2.5(1-4)$ & $2.3(1-4)$ \\
Residual LES pressure before PD, mm Hg & $23.9(15.7-44.4)$ & $28.5(16.1-55.7)$ & $25.4(17.2-33.9)$ \\
PD pressure, psi & $9.4(7-15)$ & $13.0(6-19)$ & $9.6(9-15)$ \\
6-month residual LES pressure after PD, mm Hg & $7.9(1.6-10.5)$ & $13.0(6.5-18.5)$ & $16.1(8.8-21.6)$ \\
\hline
\end{tabular}

Data are expressed as median (interquartile range). ${ }^{*} \mathrm{p}<0.05$ versus type I.

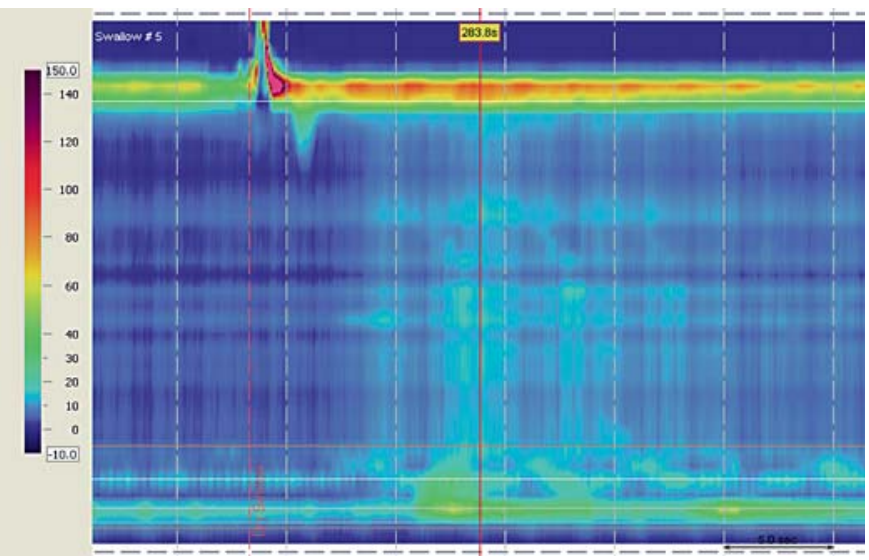

Fig. 1. Type I achalasia: there is no distal esophageal pressurization greater than $30 \mathrm{~mm} \mathrm{Hg}$ in more than 8 of the 10 test swallows.

\section{Results}

\section{Demographic Data}

Twenty-five patients were diagnosed with primary achalasia with HRM and were treated by PD in our hospital. The demographic characteristics of the 25 patients with achalasia according to the new classification of achalasia are summarized in table 1 . The age of those with type III achalasia was significantly older than those with type I achalasia. However, there were no statistical differences in other factors among the patients with the 3 types of achalasia.

\section{Success of First PD}

After the first treatment of $\mathrm{PD}$, remission occurred in 24 out of $25(96.0 \%)$ patients after 6 months, and in 19 out of $25(76.0 \%)$ patients after 12 months (fig. 4 ). The success

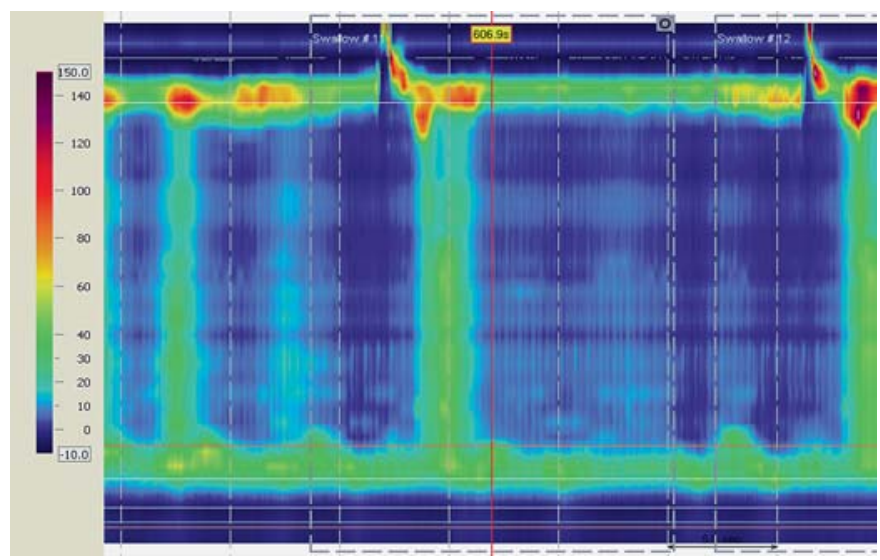

Fig. 2. Type II achalasia: at least 2 test swallows were associated with panesophageal pressurization greater than $30 \mathrm{~mm} \mathrm{Hg}$.

ratio of PD after 6 and 12 months according to the new classification of achalasia is shown in table 2. Patients with type III achalasia tended to require further PD compared to those with type I and type II achalasia. The median duration of follow-up was 31.1 months. Twentythree out of $25(92.0 \%)$ patients required 1 dilatation and 2 (8\%) patients needed 2 dilatations to achieve remission in the first PD. The 2 patients who needed 2 dilatations in the first PD were young females aged 29 and 39 years old.

\section{Predictive Factors Associated with the Success of PD}

The median age in the successful group was significantly greater than that of the failure group (47.1 vs. 37.0 years, $\mathrm{p}<0.05$ ). The median residual LES pressure 6 months after the first PD in the successful group was significantly lower than that of the failure group (9.0 vs. 15.5 


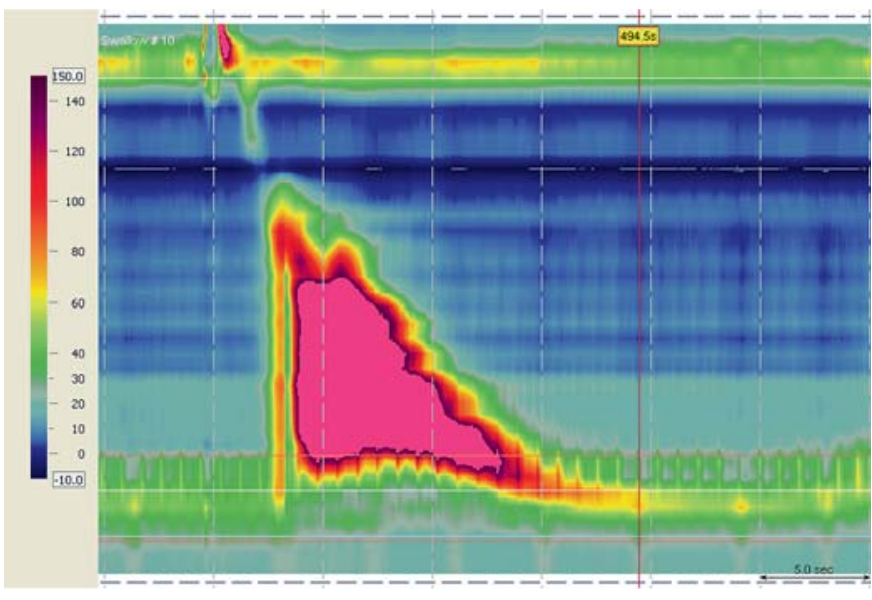

Fig. 3. Type III achalasia: these patients had 2 or more spastic contractions with or without periods of compartmentalized pressurization.

$\mathrm{mm} \mathrm{Hg}, \mathrm{p}<0.05)$. However, there were no differences in the other factors between the groups (table 3 ). Old age and lower residual pressures 6 months after PD were the factors associated with a better response to PD. Setting the cutoff value at 40 years of age resulted in an $85.7 \%$ cumulative success of $\mathrm{PD}$ in the $>40$ age group, but only $46.7 \%$ in the $<40$ age group. Also, setting the cutoff value at 15 $\mathrm{mm} \mathrm{Hg}$ of residual pressure at 6 months after PD resulted in a $90.0 \%$ cumulative success of $\mathrm{PD}$ in the $<15 \mathrm{~mm} \mathrm{Hg}$ group, but only $20.0 \%$ in the $>15 \mathrm{~mm} \mathrm{Hg}$ group.

\section{Complications}

There were no perforations related to PD. Two of the $25(8 \%)$ patients had reflux esophagitis of grade A or B of the Los Angeles classification, and the esophagitis responded well to standard doses of proton pump inhibitors.

\section{Discussion}

Achalasia is a chronic condition with a broad spectrum of expressions. Treatment is palliative and followup is lifelong. Therefore, most gastroenterologists prefer $\mathrm{PD}$ as the first line of therapy. In a recent meta-analysis that studied the pooled results of 15 articles and 1,065 patients who underwent $\mathrm{PD}$, the rates of symptom relief were $73.8 \%$ at 6 months, $68 \%$ at 12 months and $58 \%$ beyond 36 months, and $25 \%$ of patients required repeat dilatation [10]. These were outcomes that were consistent with our study.

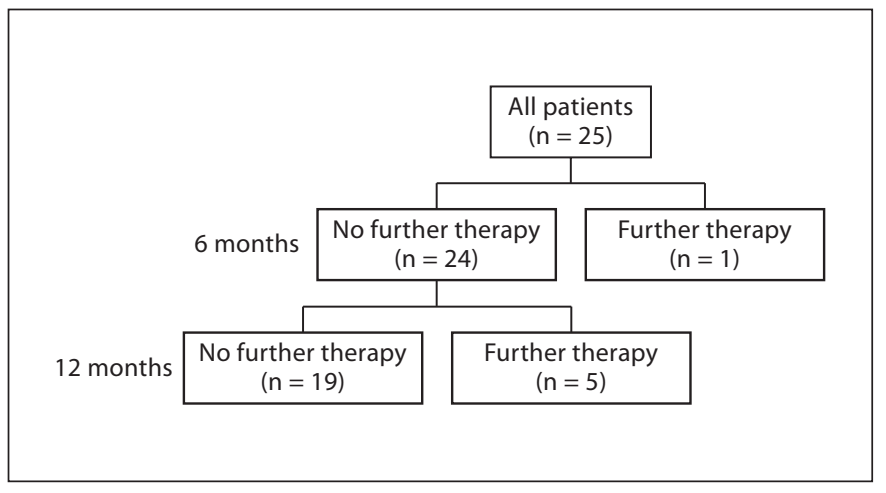

Fig. 4. One patient experienced recurrence within 6 months and 5 patients experienced recurrence within 12 months of the first PD. The success ratio was 96.0 and $76.0 \% 6$ and 12 months after $\mathrm{PD}$, respectively.

Table 2. Success ratio of PD after 6 and 12 months according to the new classification of achalasia

\begin{tabular}{rlll}
\hline \multicolumn{4}{c}{ Success ratio, \% } \\
\cline { 2 - 4 } & $\begin{array}{l}\text { type I } \\
(\mathrm{n}=6)\end{array}$ & $\begin{array}{l}\text { type II } \\
(\mathrm{n}=15)\end{array}$ & $\begin{array}{l}\text { type III } \\
(\mathrm{n}=4)\end{array}$ \\
\hline 6 months & $100(6 / 6)$ & $100(15 / 15)$ & $66.6(3 / 4)$ \\
12 months & $83.3(5 / 6)$ & $80.0(12 / 15)$ & $50.0(2 / 4)$ \\
\hline
\end{tabular}

It is important to be able to predict the response to $\mathrm{PD}$. Age was thought to be the only factor that determined the response to dilation $[4,11,12]$. Howard et al. [13] reported that a young age at diagnosis and an increased esophageal width on barium swallows predicted a worse outcome. Our study demonstrated that old age ( $>40$ years) and lower residual LES pressures $(<15 \mathrm{~mm} \mathrm{Hg})$ at 6 months after PD were the factors that were associated with a better response to PD. Regarding the age factor, our results were consistent with other published data showing that older patients respond favorably to PD compared to younger patients [14]. However, it is well known that LES pressure decreases in the short term after PD [5, 15, 16]. Eckardt et al. [12] reported that LES pressures less than $10 \mathrm{~mm} \mathrm{Hg}$ after PD was the most significant predictive factor of favorable long-term outcomes. In addition, Penagini [17] reported that in the patients who needed further treatment the LES pressure 3 months after the first treatment was $10 \mathrm{~mm} \mathrm{Hg}$ or more, whereas the pres- 
Table 3. Predictive factors associated with the success of PD

\begin{tabular}{lccc}
\hline & Success $(\mathrm{n}=19)$ & Failure $(\mathrm{n}=6)$ & $\mathrm{p}$ value \\
\hline Age, years & $47.1 \pm 18.7$ & $37.0 \pm 12.8$ & $<0.05$ \\
Gender (male/female) & $9 / 10$ & $3 / 3$ & n.s. \\
Duration of symptoms, years & $5.9 \pm 2.3$ & $6.7 \pm 3.4$ & n.s. \\
Residual LES pressure before PD, mm Hg & $20.2 \pm 11.8$ & $24.3 \pm 5.8$ & n.s. \\
PD pressure, psi & $11.5 \pm 4.9$ & $10.0 \pm 2.7$ & n.s. \\
6-month residual LES pressure after PD, mm Hg & $9.0 \pm 3.7$ & $15.5 \pm 5.7$ & $<0.05$ \\
\hline
\end{tabular}

Data are expressed as median $\pm \mathrm{SE}$.

sure in all of the patients who underwent only one dilatation was less than $10 \mathrm{~mm} \mathrm{Hg}$. However, in these reports, the method used for the manometry was the infusion pump method. There have been few studies that have evaluated LES pressures after PD using HRM, which is the most accurate method for evaluating LES pressure. We examined LES pressures using HRM and, therefore, our study differed from the previous studies.

Pandolfino et al. [8] proposed a new classification of achalasia using HRM in which impaired LES relaxation was defined as greater than $15 \mathrm{~mm} \mathrm{Hg}$. In addition, they categorized achalasia into 3 subtypes that were distinct in terms of their responsiveness to PD therapies. They reported that patients with type III achalasia were resistant to $\mathrm{PD}$, which was consistent with our study. In our study the age of patients with type III achalasia was significantly older than those with type I achalasia; however, the success ratio of PD in type III achalasia patients was lower than in those of type I achalasia. We think the high residual LES pressures in type III achalasia 6 months after PD compared to those of type I achalasia is the reason for the low clinical remission in type III achalasia. Our data showed that patients who developed clinical relapses after reaching clinical remission had residual LES pressures that were higher than $15 \mathrm{~mm} \mathrm{Hg} 6$ months after the first dilatation. On the contrary, residual LES pressures before dilatation and PD pressures did not discriminate patients who developed clinical relapses after reaching remission from those who did not. We think it is important to decrease the residual LES pressure to less than 15 $\mathrm{mm} \mathrm{Hg}$ for remission of symptoms. Therefore, the definition of impaired LES relaxation as that greater than 15 $\mathrm{mm} \mathrm{Hg}$, which was suggested by Pandolfino et al. [8], is appropriate.

Previous studies have shown that unsatisfactory first dilation results were always evident within 1 year. Actu- ally, we found that most of the relapses occurred early after the first dilation (within 1 year). Therefore, we think that the patients should be carefully followed up in the first year after PD. Bravi et al. [18] reported a strategy that includes sessions of PD until clinical remission and a standardized follow-up result in a low need for further dilatations and a high rate of clinical success over the long term. Furthermore, a careful follow-up during the first year after treatment allows for the rapid identification of patients who do not respond to endoscopic therapy and a chance to offer them surgery [18]. A long-term strategy of standardized follow-up assessments is important because it is known that, during on-demand follow-up, patients may underestimate their symptoms, have a poor quality of life and possible complications, and eventually come back to the physician when the esophagus is dilated and treatment is less effective [6]. Esophageal manometry by HRM may be a useful objective for evaluating achalasia treatment and for deciding on the need for further dilations.

Esophageal perforation is the most serious complication of PD. In our study, no patients had an esophageal perforation during treatment with $\mathrm{PD}$. In the meta-analysis of the series, the mean perforation rate was 1.6\% [10]. The most frequent complication was gastroesophageal reflux disease. In our study, 2 of the 25 (8\%) patients had reflux esophagitis of grades A or B of the Los Angeles classification, and responded well to standard doses of proton pump inhibitors.

In conclusion, this study suggests that an initial PD is a reasonable approach as first-line therapy in patients with achalasia. Good predictors of success are old age (more than 40 years) and residual pressures less than 15 $\mathrm{mm} \mathrm{Hg} 6$ months after PD. Therefore, the practice of serial PD for all patients with achalasia may need to be modified for young men. In this group, initial therapy 
with a larger balloon size or surgical esophagomyotomy might reduce the likelihood of repeated dilation. Esophageal manometry by HRM may be a useful objective test for evaluating achalasia treatment and possibly for deciding on the need for further dilations.

\section{Disclosure Statement}

None.

\section{References}

1 Cohen S, Fisher R, Tuch A, et al: The site of denervation in achalasia. Gut 1972;13:556558.

2 Mayberry JF: Epidemiology and demographics of achalasia. Gastrointest Endosc Clini N Am 2001;11:235-248.

-3 Vaezi MF, Richter JE: Current therapies for achalasia. J Clin Gastroenterol 1998;27:2135.

-4 Tanaka Y, Iwakiri K, Kawami N, Sano Hiroshi, Umezawa M, Kotoyori M, Hoshihara Y, Nomura T, Miyashita M, Sakamoto C: Predictor of a better outcome of pneumatic dilatation in patients with primary achalasia. J Gastroenterol 2010;45:153-158.

5 Eckardt VF, Aignhrr C, Bernhard G: Predictors of outcome in patients with achalasia treated by pneumatic dilation. Gastroenterology 1992;103:1732-1738.

-6 West RL, Hirsch DP, Bartelsman JFWM, Borst J, Ferwerda G, Tytgat GNJ, Boeckxstaens GE: Long term results of pneumatic dilatation in achalasia followed for more than 5 years. Am J Gastroenterol 2002;97: 1346-1351.

7 Torbey CF, Achkar E, Rice TW, Baker M, Rchter JE: Long-term outcome of achalasia treatment: the need for closer follow up. J Clin Gastroenterol 1999;28:125-130.
8 Pandolfino JP, Kwiatek MA, Nealis T, Bulsiewicz W, Post J, Kahrias PJ: Achalasia: a new clinically relevant classification by high-resolution manometry. Gastroenterol 2008;135: 1526-1533.

$\checkmark 9$ Ghosh SK, Pandolfino JE, Rice J, Clarke JO, Kwiatek M, Kahrilas PJ: Impaired deglutitive EGJ relaxation in clinical esophageal manometry: a quantitative analysis of $400 \mathrm{pa}$ tients and 75 controls. Am J Physiol Gastrointest Liver Physiol 2007;293:G878-G885.

10 Campos GM, Vittinghoff E, Rabl C, Takata M, Gadenstatter M, Lin F, Ciovica R: Endoscopic and surgical treatments for achalasia: a systematic review and meta-analysis. Ann Surg 2009;249:45-57.

11 Farhoomand K, Connor JT, Richter JE, Achkar E, Vaezi MF: Predictors of outcome of pneumatic dilation in achalasia. Clin Gastroenterol Hepatol 2004;2:389-394.

12 Eckardt VF, Gockel I, Bernbard G: Pneumatic dilation for achalasia: late results of a prospective follow up investigation. Gut 2004; 53:629-633.

13 Howard JM, Mongan AM, Manning BJ, Byrne P, Lawler P, Ravi N, Reynolds JV: Outcomes in achalasia from a surgical unit where pneumatic dilation is first-line therapy. Dis Esophagus 2010;23:465-472.
14 Vela MF, Richter JE, Khandwala F, Blackstone $\mathrm{EH}$, Wachsberger D, Baker ME, Rice TW: The long-term efficacy of pneumatic dilatation and Heller myotomy for the treatment of achalasia. Clin Gastroenterol Hepatol 2006;4:580-587.

15 Wehrmann T, Jacobi V, Jung M, Lembcke B, Caspary W: Pneumatic dilation in achalasia with a low-compliance balloon: results of a 5 -year prospective evaluation. Gastrointestinal Endosc 1995;42:31-36.

16 Kadakia S, Wong R: Graded pneumatic dilatation using Rigiflex achalasia dilators in patients with primary esophageal achalasia. Am J Gastroenterol 1993;88:34-38.

17 Penagini R, Cantu P, Mangano M, Colombo P, Bianchi PA: Long-term effect of pneumatic dilation on symptoms and lower oesophageal sphincter pressure in achalasia. Scand J Gastroenterol 2002;37:380-384.

18 Bravi I, Nicita MT, Duca P, Grigolon A, Cantu P, Caparello C, Penagini R: A pneumatic dilation strategy in achalasia: prospective outcome and effects on oesophageal motor function in the long term. Aliment Pharmacol Ther 2010;31:658-665. 